\title{
Anti-aging Properties of Conditioned Media of Epidermal Progenitor Cells Derived from Mesenchymal Stem Cells
}

\author{
Su Ji Sohn · Ji Min Yu - Eun Young Lee · You Jin Nam • \\ Jinwan Kim · Sukho Kang • Dong Hyun Kim • Aeri Kim • \\ Sangjin Kang (D)
}

Received: December 26, 2017 / Published online: March 2, 2018

(c) The Author(s) 2018. This article is an open access publication

\begin{abstract}
Introduction: Reduced number and activities of epidermal stem cells are related to the features of photoaged skin. It was reported that conditioned media from various stem cell cultures are capable of improving the signs of cutaneous aging. This work was performed to
\end{abstract}

Enhanced content To view enhanced content for this article go to https://doi.org/10.6084/m9.figshare. 5882878 .

Su Ji Sohn and Ji Min Yu contributed equally to this study.

Electronic supplementary material The online version of this article (https://doi.org/10.1007/s13555018-0229-2) contains supplementary material, which is available to authorized users.

S. J. Sohn · E. Y. Lee · Y. J. Nam · S. Kang Department of Biotechnology, CHA University, Seongnam, Republic of Korea

J. M. Yu

R\&D Division, CHA Biotech, Co. Ltd., Seongnam, Republic of Korea

J. Kim · S. Kang $(\bowtie)$

Chabio F\&C, Seongnam, Republic of Korea

e-mail: sjkang@cha.ac.kr

S. Kang

Department of Obstetrics and Gynecology, CHA

Bundang Medical Center, CHA University,

Seongnam, Republic of Korea establish epidermal progenitor cells derived from mesenchymal stem cells, and to evaluate the anti-aging efficacy of its conditioned media. Methods: Epidermal progenitor cell culture was established by differentiation from mesenchymal stem cells, and its conditioned medium (EPC-CM) was prepared. Normal human dermal fibroblasts were exposed to hydrogen peroxide and the protective effects of EPC-CM were investigated, monitoring intracellular reactive oxygen species (ROS), cellular defense enzymes, collagen biosynthesis, and mitogen-associated protein kinase (MAPK) signaling. Anti-aging efficacy of cosmetic essence (5\% EPC-CM) was evaluated by a clinical test with 25 Korean women aged between 29 and 69 .

Results: Hydrogen peroxide hindered proliferation of fibroblasts and increased the levels of intracellular ROS. Pretreatment of EPC-CM

\section{H. Kim}

Department of Dermatology, CHA Bundang Medical Center, CHA University, Seongnam, Republic of Korea
A. Kim
College of Pharmacy, CHA University, Seongnam, Republic of Korea 
protected fibroblasts from oxidative stress as shown by accelerated proliferation and reduced ROS generation. EPC-CM effectively prevented hydrogen peroxide-induced alterations of the activities, as well as mRNA and protein levels, of antioxidative enzymes, such as superoxide dismutase, catalase, and glutathione peroxidase. Reduced type I collagen biosynthesis and stimulated phosphorylation of MAPK signaling proteins, induced by oxidative damage, were also prevented by EPC-CM. In clinical study, wrinkle, depression, and skin texture were improved by the topical application of a formulation containing 5\% EPC-CM within 4 weeks.

Conclusion: Epidermal progenitor cell culture was established, and its conditioned medium was developed for anti-aging therapy. EPC-CM improved signs of skin aging in clinical study, possibly via activation of cellular the defense system, as supported by in vitro results.

Keywords: Anti-aging; Epidermal progenitor cell-conditioned media; Oxidative stress

\section{INTRODUCTION}

Skin photoaging describes the changes in older skin that has been habitually exposed to the sun; such skin is characterized by epidermal thickening, coarse wrinkling, actinic elastosis, and reduced collagen content in the papillary dermis. The effects of chronic sun damage are added onto intrinsic aging changes [1]. Normal skin homeostasis is maintained by epidermal stem cells which produce differentiating epidermal cells [2,3]. Stem cells also signal to other cells within the skin via both cell-cell contact and diffusible factors [2, 4, 5]. Kwon et al. reported that the number of keratinocyte stem cells may be lower in photoaged skin than in chronologically aged skin [6], suggesting that reduced number and function of epidermal stem cells could be a factor responsible for the changes observed in photoaged skin.

Exposure to UV radiation increases the production of intracellular reactive oxygen species (ROS), which leads to oxidative stress and photoaging of the skin [7]. ROS are composed of superoxide anion radical $\left(\mathrm{O}_{2}^{\bullet-}\right)$, hydrogen peroxide $\left(\mathrm{H}_{2} \mathrm{O}_{2}\right)$, hydroxyl radical $\left(\mathrm{OH}^{\bullet}\right)$, singlet oxygen $\left({ }^{1} \mathrm{O}_{2}\right)$, and other oxygen-centered reactive materials $[8,9]$. Cellular antioxidative defense enzymes and small molecule antioxidants scavenge ROS or repair damage produced by the action of oxidative stress. Key members of the antioxidant defense system include catalase, superoxide dismutase (SOD), and glutathione peroxidase (GPx) [10]. SOD catalyzes the dismutation of superoxide radical into hydrogen peroxide and molecular oxygen, while catalase and GPx remove hydrogen peroxide $[7,11]$. Activities of defense enzymes are reduced by UV exposure or oxidative damage [12-14].

Recently, conditioned media of various stem cells, mostly mesenchymal stem cells, were reported to possess anti-aging properties and used in anti-aging formulations [15-17]. Media collected from epidermal stem cell culture could be expected to exhibit better effects on aged skin than those from other stem cells. However, it is difficult to establish a stable cell line and to produce conditioned media with reproducible quality, because epidermal stem cells readily undergo terminal differentiation in culture [18-20].

In this study, epidermal progenitor cells were derived from mesenchymal stem cells, and the anti-aging properties of the conditioned medium (EPC-CM) were investigated in human dermal fibroblasts, and the efficacy was evaluated in a clinical study.

\section{METHODS}

\section{Differentiation of Mesenchymal Stem Cells into Epidermal Progenitor Cells (EPCs)}

Human mesenchymal stem cells (hMSCs) were provided by CHA Biotech, Co. Ltd. (Seongnam, Korea). All the manufacturing and product testing procedures for the generation of hMSCs were performed under good manufacturing practice conditions. Preparation and characterization of cells have been described previously [21]. 
To initiate differentiation, hMSCs were plated at a density of about 1000 cells $/ \mathrm{cm}^{2}$ into a T175 culture flask with complete culture medium. After 2-3 days, the medium was removed and washed with PBS. The cells were replaced with differentiation medium, consisting of Dulbecco's Modified Eagle Medium/Nutrient Mixture F-12 (DMEM/F12, Life Technologies, Carlsbad, CA, USA), 10\% fetal bovine serum (Life Technologies), $0.3 \mu \mathrm{M}$ ascorbic acid (Sigma Aldrich, Louis, MO, USA), and $0.5 \mu \mathrm{g} / \mathrm{mL}$ hydrocortisone (Sigma Aldrich). After 1021 days from the initial plating, when the cells acquired a rounded or polygonal shape, the expression of basal keratinocyte marker, cytokeratin 14 was assessed.

\section{Conditioned Media (CM) Preparation}

hMSCs were differentiated to EPCs for 1021 days in a T175 culture flask, then washed extensively with PBS, and replenished with $20 \mathrm{~mL}$ chemically defined media (MEM alpha without phenol red, choline chloride, vitamin B12, and sodium phosphate monobasic, supplemented with lipoic acid, adenosine, cyanocobalamin, cytidine, guanosine, DL-alphalipoic acid, and uridine for clinical use) for $48-72 \mathrm{~h}$ prior to harvesting the media for further experimentation (EPC-CM). Conditioned medium was also collected from undifferentiated hMSC for comparison (MSC-CM). Collected media samples were centrifuged at $3000 \mathrm{rpm}$ for $10 \mathrm{~min}$ to remove cell debris, and filtered through a $0.22-\mu \mathrm{m}$ filter. Then, the medium was concentrated by a factor of 50 using centrifugal filter units with 3-kDa cutoff (Millipore, Billerica, MA, USA) following the manufacturer's instructions. All the concentrated $\mathrm{CM}$ was kept at $-80^{\circ} \mathrm{C}$ until use.

\section{RNA Extraction from Differentiated Cells and Real-Time RT-PCR Analysis}

Total RNA from differentiated cells was extracted with RNeasy Mini kit (Qiagen, Hilden, Germany) and about $0.1-1 \mu \mathrm{g}$ of total RNA per sample was used to synthesize double-stranded cDNA by reverse transcription (SuperScript III;
Life Technologies). Real-time PCR was performed in triplicate for human GAPDH, Krt10, Krt14, or involucrin using SYBR Green Expression Assays (Life Technologies). Primers used in this study were as follows: 5'-CCA GGT CCA AGA CAT TCA AC-3' and 5'-ACT GCG GGT GGT TAT TTA TG-3' to amplify Involucrin mRNA, 5'-ACT ACT CTT CCT CCC GCA GT-3' and $5^{\prime}$-TGA GCT AAA TCC TCC ACC AA-3' to amplify cytokeratin $10 \mathrm{mRNA}$, and $5^{\prime}$-GAG CAG CAG AAC CAG GAG T-3' and 5'-GAG AAC TGG GAG GAGGAG AG-3' to amplify cytokeratin 14 mRNA. Real-time amplification was performed with SYBR Green PCR Master Mix (Life Technologies) and analyzed on a 7500 real-time PCR system (Life Technologies). For assays, the reactions were incubated at $50^{\circ} \mathrm{C}$ for $2 \mathrm{~min}$ followed by $95^{\circ} \mathrm{C}$ for $10 \mathrm{~min}$, and then 40 thermal cycles (at $95{ }^{\circ} \mathrm{C}$ for $15 \mathrm{~s}$ followed by $60{ }^{\circ} \mathrm{C}$ for $1 \mathrm{~min}$ ) were performed. Data were analyzed with Sequence Detection Software V2.3 (Life Technologies) and relative quantities (RQs) were calculated with a comparative CT method using RQ Manager V1.2 (Life Technologies).

\section{Immunocytochemistry of EPC}

hMSCs were plated at initial densities of about 1000 cells $/ \mathrm{cm}^{2}$ in a slide chamber (LAB-TEK 11 chamber slide; Nalgene Nunc International, Rochester, NY, USA). After differentiation for 21 days, the cultures were rinsed with PBS and fixed in 4\% paraformaldehyde in PBS for $20 \mathrm{~min}$ at room temperature. The slide chambers were incubated for $24 \mathrm{~h}$ at $4{ }^{\circ} \mathrm{C}$ with primary antibody/cytokeratin 14 (1:100; Abcam, Cambridge, UK). The slides were washed three times for 5 min with PBS and incubated for $1 \mathrm{~h}$ at room temperature with secondary antibody: Alexa488 (1:1000; Molecular Probes, Carlsbad, CA, USA). The cells were counterstained with DAPI solution (Life Technologies). Slides were evaluated by epifluorescence (Eclipse Ti; Nikon, Tokyo, Japan) using a $100 \times$ objective. Images were analyzed using software (Diagnostic Instruments Inc., Sterling Heights, MI, USA). 


\section{Cytokine Secretion Profiling by Cytokine Antibody Array}

The EPC-CM was analyzed for the presence of cytokines, chemokines, and growth factors by performing a semiquantitative human growth factor antibody based array (RayBio, Norcross, GA, USA). The experiment was performed as per the manufacturer's instructions and chemiluminescence was recorded using an Image Quant LAS 4000 (GE Healthcare, Buckinghamshire, UK). The data was analyzed using Image J software. The relative intensities of individual growth factors were calculated as arbitrary units after background correction and normalized to control media blot intensities.

\section{Fibroblast Culture}

Normal human dermal fibroblast from neonatal tissue (NHDF) was purchased from Lonza (Basel, Switzerland). The NHDF cells were grown in Dulbecco's Modified Eagle's Medium (Hyclone Laboratories, Inc., Logan, UT, USA) containing $10 \%$ fetal bovine serum (Hyclone Laboratories, Inc.) and 1\% Antibiotic-Antimycotic (Gibco, Waltham, MA, USA) at $37^{\circ} \mathrm{C}$ in a humidified atmosphere of a $5 \% \mathrm{CO}_{2}$ incubator.

\section{Cell Proliferation}

Cell viability was measured using an EZ-Cytox assay kit (Daeil Lab Service, Seoul, Korea) according to the manufacturer's instruction. NHDF cells were pretreated with or without each conditioned medium $(1 \times)$ for $24 \mathrm{~h}$, followed by exposure to $600 \mu \mathrm{M} \mathrm{H}_{2} \mathrm{O}_{2}$ for $24 \mathrm{~h}$. Medium was replaced with serum-free media with $20 \mu \mathrm{L} /$ well of EZ-Cytox and incubated for $2 \mathrm{~h}$ before absorbance was measured at $450 \mathrm{~nm}$ by a Synergy H1 microplate reader (BioTek, Winooski, VT, USA). The relative cell proliferation was expressed as fold changes compared with the control group.

\section{Measurement of Intracellular Reactive Oxygen Species (ROS) Formation}

NHDF cells were pretreated with each conditioned medium $(1 \times)$ for $24 \mathrm{~h}$, then exposed to $600 \mu \mathrm{M} \mathrm{H} \mathrm{H}_{2} \mathrm{O}_{2}$ in PBS containing $0.1 \%$ serum and $10 \mu \mathrm{M} \quad 2^{\prime}, 7^{\prime}$-dichlorodihydrofluorescein diacetate acetyl ester $\left(\mathrm{H}_{2}\right.$ DCFDA, Molecular Probes) for $15 \mathrm{~min}$. Cells were lysed with $1 \mathrm{~N}$ $\mathrm{NaOH}$ and transferred to 96-well black plates for analysis. ROS formation was determined as a measure of DCF fluorescence intensity at an excitation wavelength of $485 \mathrm{~nm}$ and an emission wavelength of $528 \mathrm{~nm}$ by a Synergy H1 microplate reader.

\section{Activities of Antioxidative Defense Enzymes}

The activities of catalase, SOD, and GPx were measured using catalase activity assay kit (Cayman Chemical, Ann Arbor, MI, USA), superoxide dismutase activity assay kit (Cayman Chemical), and glutathione peroxidase activity kit (Abcam), respectively. NHDF cells were seeded in $100-\mathrm{mm}$ dishes at a density of $5 \times 10^{5}$ cells. Cells were pretreated or not pretreated with each conditioned medium $(1 \times)$ for $24 \mathrm{~h}$, followed by exposure to $600 \mu \mathrm{M} \mathrm{H}_{2} \mathrm{O}_{2}$ for $2 \mathrm{~h}$. The reaction was processed according to the manufacturer's protocol. Activities of catalase, superoxide dismutase, and glutathione peroxidase were measured at 540, 440, and $340 \mathrm{~nm}$, respectively.

\section{Type I Procollagen C-terminal Peptide (PICP) Assay}

Type I procollagen C-terminal peptide was measured using PICP EIA kit (Takara, Kyoto, Japan). NHDF cells were seeded in 6-well plates at a density of $5 \times 10^{4}$ cells/well. Cells were treated with or without each conditioned medium $(1 \times)$ for $24 \mathrm{~h}$, followed by exposure to $600 \mu \mathrm{M} \mathrm{H} \mathrm{H}_{2} \mathrm{O}_{2}$ for $24 \mathrm{~h}$, Afterwards, the supernatant of each sample was collected. The reaction was processed according to the manufacturer's protocol. Briefly, $100 \mu \mathrm{L}$ of peroxidase conjugated antibody solution and $20 \mu \mathrm{L}$ 
of sample supernatant or standard solution were incubated for $3 \mathrm{~h}$ at $37^{\circ} \mathrm{C}$. After wash, $100 \mu \mathrm{L}$ substrate solution of peroxidase was added and incubated at room temperature for $15 \mathrm{~min}$. The reaction was then quenched by adding stop solution and absorbance was measured at $450 \mathrm{~nm}$ using a Synergy H1 microplate reader.

\section{Semiquantitative Reverse Transcription Polymerase Chain Reaction (RT-PCR)}

The total RNA was extracted with Nucleospin RNA/protein kit (Macherey-Nagel, Dueren, Germany). Double-stranded cDNA was synthesized from $2 \mu \mathrm{g}$ of total RNA by reverse transcription. PCR was performed with $10 \mathrm{pM}$ of specific primers: 5'-CGT GCT GAA TGA GGA ACA GA-3' and 5'-AGT CAG GGT GGA CCT CAG TG-3' to amplify catalase mRNA, 5'-CTG AAG GCC TGC ATG GAT TC- $3^{\prime}$ and $5^{\prime}$-CCA AGT CTC CAA CAT GCC TCT C-3' to amplify SOD 1 mRNA, 5'-ACG ATG TTG CCT GGA ACT TT- ${ }^{\prime}$ and $5^{\prime}$-GAT GTC AGG CTC GAT GTC AA$3^{\prime}$ to amplify GPx mRNA, 5'-GCT GTC TTA TGG CTA TGA TGA G-3' and 5'-CTT CCC CAT CAT CTC CAT TC-3' to amplify type I procollagen mRNA, and 5'-GCG TGA CAT TAA GGA GC-3'

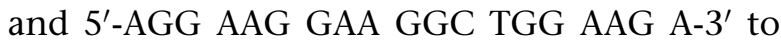
amplify actin mRNA. PCR products were separated on $2 \%$ agarose gel and visualized.

\section{Western Blot Analysis}

Proteins were extracted from cells using Nucleospin RNA/protein kit (Macherey-Nagel). Equivalent amounts of total cell lysate were subjected to SDS-PAGE. Western blot was done with antibodies for $\beta$-actin (1:5000; Abcam), cytokeratin 14 (1:1000, Abcam), catalase (1:2000; Abcam), SOD 1 (1:2000; Abcam), GPx (1:1000; Abcam), Erk (1:1000; Cell Signaling Technology, MA, USA), phospho-Erk (1:2000; Cell Signaling Technology), JNK (1:1000; Cell Signaling Technology), phospho-JNK (1:2000; Abcam), c-Jun (1:1000; Cell Signaling Technology), phospho-c-Jun (1:1000; Cell Signaling Technology), p38 (1:1000; Cell Signaling Technology), phospho-p38 (1:1000; Cell Signaling Technology), Akt (1:5000; Cell Signaling
Technology), phospho-Akt (1:1000; Cell Signaling Technology), or GAPDH $(1: 10,000$; AbFrontier, Seoul, Korea). Bound primary antibodies were detected with conjugated secondary antibodies, observed by enhanced chemiluminescence using Clarity $^{\mathrm{TM}}$ Western ECL (Bio-Rad Laboratories, Hercules, CA, USA), photographed and quantified with Gel-doc Image Lab (Bio-Rad Laboratories).

\section{Clinical Study}

All procedures performed in studies involving human participants were in accordance with the ethical standards of the institutional research committee (IRB File No. 2016-04-039, Bundang CHA Hospital, Korea) and with the 1964 Declaration of Helsinki and its later amendments or comparable ethical standards. Informed consent was obtained from all individual participants included in the study.

Twenty-five female volunteers aged 2969 years with mild to moderate wrinkles were included. Cosmetic essence with 5\% EPC-CM was applied twice a day on both sides of the face for 4 weeks. Before application, baseline was evaluated for Fitzpatrick skin type, crow's feet scale [22], physician global assessment scale [23], and wrinkle index by ANTERA $3 \mathrm{D}^{\circledR}$ (Miravex, Dublin, Ireland). The baseline characteristics of subjects are shown in Table 1. Two dermatologists performed the scale evaluations. The wrinkle index of ANTERA 3D ${ }^{\circledR}$ included wrinkle depth (crow's feet), skin surface (depression, cheek), and skin texture ( $\mathrm{Ra}$, cheek).

Table 1 Characteristics of subjects

\begin{tabular}{ll}
\hline & $\begin{array}{l}\text { Volunteers } \\
(\boldsymbol{n}=\mathbf{2 5})\end{array}$ \\
\hline Age, mean \pm SD & $49.7 \pm 13.2$ \\
Fitzpatrick skin type scale (1-5), & $3.92 \pm 0.17$ \\
$\quad$ mean \pm SD & \\
Crow's feet scale $(0-4)$, mean \pm SD & $2.38 \pm 1.2$ \\
PGA $(0-4)$, mean \pm SD & $2.15 \pm 1.3$ \\
\hline
\end{tabular}

$P G A$ physician global assessment 
The wrinkle index was measured by specifying the area where the movement of the facial muscle was minimized. The wrinkle depth is measured at the deepest point in the specified area, and the skin surface reflects the volume of the entire depressed area at the specific area. Skin texture shows the degree of surface roughness as Ra value of ANTERA $3 \mathrm{D}^{\circledR}$ 's own program index. The Ra value is proportional to the degree of skin roughness.

\section{Statistical Analysis}

Experimental results are represented as the mean values \pm standard deviations. Statistical analyses were performed with IBM SPSS Statistics ver. 24.0 (IBM Co., Armonk, NY, USA). The statistical evaluation of the in vitro data was based on one-way ANOVA and Dunnett's test analysis of variance for means. The paired $t$ test was applied to assess changes in the depth, depression, and texture small score after intervention compared with baseline. Differences were considered significant at $p<0.05$.

\section{RESULTS}

\section{Differentiation of hMSC into Keratinocyte Lineage}

Human mesenchymal stem cells (hMSCs) possess multipotent differentiation capabilities and are potentially a readily available and accessible source of keratinocytes [24]. In order to evaluate the differentiation efficiency of hMSC into keratinocytes, hMSCs were exposed to specific differentiation medium containing a mixture of hydrocortisone and ascorbic acid for 21 days. Morphological changes were observed after 7-10 days of cultivation. These cells were proliferating, forming an adherent monolayer, and were organized in cobblestone pattern clusters (Fig. 1a). Normal human epidermal keratinocytes also have a polygonal cobblestone shape and are very similar to the transdifferentiated MSC cells.

\section{Differentiated hMSCs Express Keratinocyte Markers}

To demonstrate the progressive epithelial determination of differentiated hMSCs, multiple specific markers for keratinocytes (cytokeratins and involucrin) were selected and evaluated using real-time PCR and Western blot analysis. There was no strong expression of keratinocyte markers at the beginning of culture, but the enhancement of keratinocyte commitment was clearly observed after 9-13 days (Fig. 1b-d). Differentiated hMSCs demonstrated gene expression profiles of the keratinocyte-defining markers similar to those of keratinocytes progenitor. Furthermore, we wanted to evaluate and quantify the percentage of MSCs differentiating into keratinocyte progenitor cells. In order to achieve this, we used cytokeratin-14 as a transdifferentiating marker since it is a marker for basal keratinocytes. As early as 21 days the positive staining for cytokeratin-14 can be observed (Fig. 1e, f). Therefore, differentiated hMSCs are considered as epidermal progenitor cells (EPCs).

\section{Cytokine Secretion Profile of EPCs}

To analyze the types and levels of the accumulated factors and cytokines released by EPCs, the conditioned medium was analyzed using human cytokine array. EPC-CM contained a broad range of soluble factors which includes cytokines, chemokines, hormones, growth factors, and endocrine and angiogenic factors (Table 2).

\section{Protective Effects Against Oxidative Stress in Cultured NHDFs}

Proliferation of normal human dermal fibroblasts was reduced by hydrogen peroxide $(600 \mu \mathrm{M})$ treatment. Reduction of cell proliferation was partially prevented by pretreatment with each conditioned medium. EPC-CM showed better protection than MSC-CM (Fig. 2a). Ascorbic acid was used as a reference antioxidant throughout in vitro experiments. 
(a)

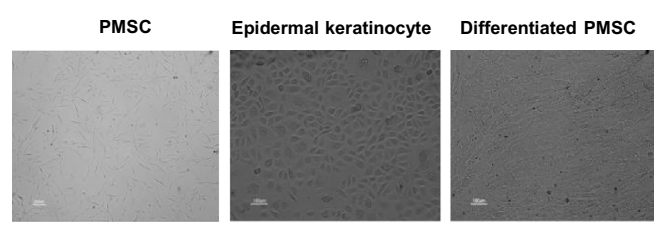

(c)

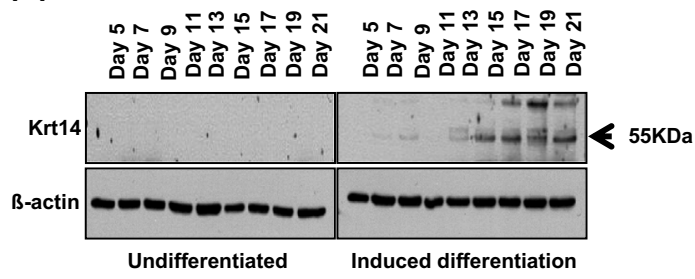

(e)

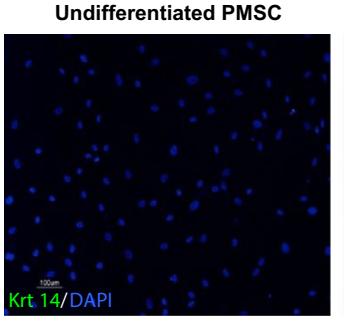

Differentiated PMSC

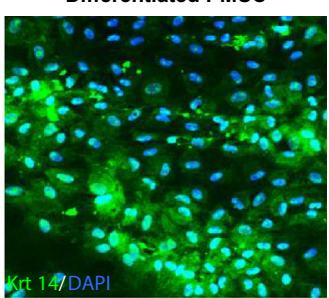

(b)

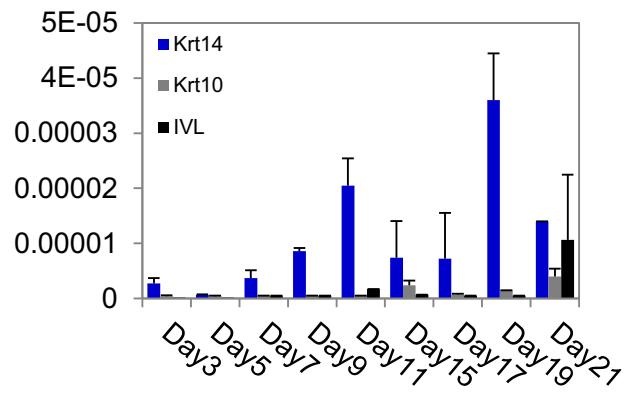

(d)

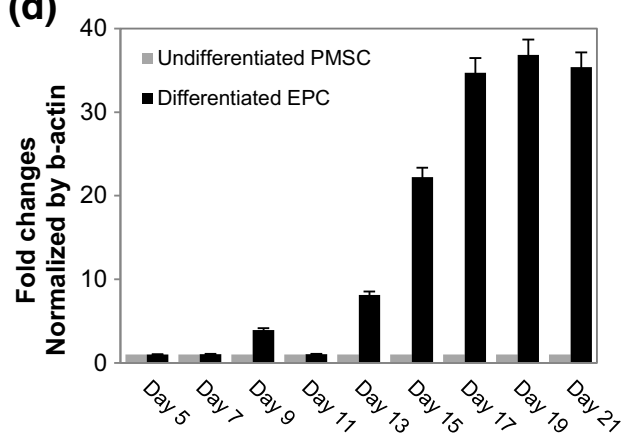

(f)

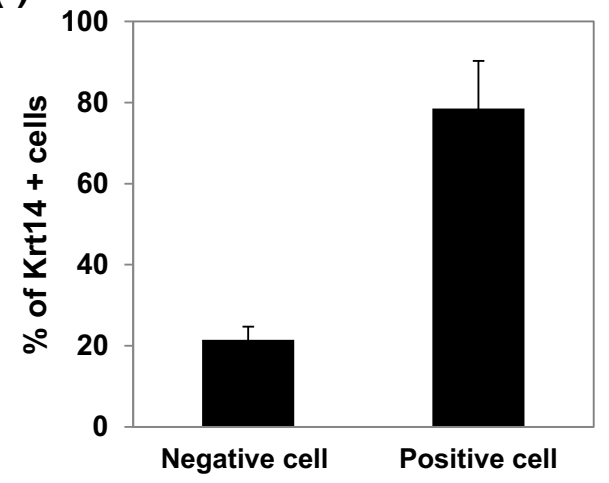

Fig. 1 hMSCs transdifferentiate into keratinocytes. a Evaluation of the morphology between hMSC, keratinocytes, and differentiated hMSC. Differentiated cells presented a polygonal morphology, characteristic for keratinocyte-like cells, and tended to cluster. b Changes in expression levels of mRNAs for keratin10, keratin14, and involucrin. c Western blot analysis for keratin 14, which confirmed

Because hydrogen peroxide is known to increase ROS levels, and elevated ROS might be responsible for the slow growth of cells, changes in intracellular ROS levels were monitored by the mRNA findings. d, e hMSCs were immunostained with anti-human cytokeratin-14. DAPI was used as counterstaining. Images demonstrate detection of cytokeratin-14 at 21 days post treatment. Data represents mean \pm SEM. Image representative of $n=3$ independent experiments

DCFDA fluorescent assay. Hydrogen peroxide induced elevation of DCF fluorescence in NHDF, which was almost completely blocked 
Table 2 List of highly upregulated cytokines on differentiation in EPCs

\begin{tabular}{|c|c|c|}
\hline Name & Full name & Signal intensity \\
\hline TSP & Thrombospondin & $5,886,805$ \\
\hline IGFBP-rp1/IGFBP-7 & Insulin & $3,970,222$ \\
\hline TIMP2 & Tissue inhibitor of metalloproteinase-2 & $3,950,540$ \\
\hline EDA-A2 & Ectodysplasin A2 & $3,938,694$ \\
\hline XEDAR & Edar and X-linked Eda-A2 receptor & $1,572,912$ \\
\hline Angiopoietin-1 & Angiopoietin-1 & $1,167,638$ \\
\hline SPARC & Secreted protein acidic and rich in cysteine & $1,020,366$ \\
\hline GDF-15 & Growth differentiation factor 15 & $1,009,469$ \\
\hline sFRP-4 & Secreted frizzled-related protein 4 & 999,123 \\
\hline GRO & Grow regulated oncogen & 963,242 \\
\hline MIP2 & Macrophage inflammatory protein 2 & 865,819 \\
\hline TIMP-1 & Tissue inhibitor of metalloproteinases 1 & 785,939 \\
\hline Latent TGF-beta bp1 & Latent TGF-beta binding protein 1 & 554,608 \\
\hline CV-2/crossveinless- 2 & Crossveinless-2 & 475,676 \\
\hline IL-6 & Interleukin 6 & 441,917 \\
\hline TMEFF $1 /$ tomoregulin-1 & Transmembrane protein with EGF-like and two follistatin-like domains 1 & 430,143 \\
\hline Nidogen-1 & Nidogen-1 & 413,625 \\
\hline Smad 4 & Mothers against decapentapiegic homolog 4 & 391,810 \\
\hline Activin $\mathrm{C}$ & Actin C & 228,189 \\
\hline IGFBP-3 & Insulin-like growth factor-binding protein 3 & 182,444 \\
\hline Thrombospondin- 2 & Thrombospondin-2 & 112,674 \\
\hline TRANCE & Tumor necrosis factor-related activation-induced cytokine & 100,065 \\
\hline Activin A & Activin A & 76,102 \\
\hline IL-15 R alpha & Interleukin-15 receptor alpha & 51,270 \\
\hline
\end{tabular}

by pretreatment with EPC-CM. The effect of MSC-CM was not significant (Fig. 2b).

\section{Activation of Antioxidative Defense Enzymes}

To investigate the mechanism underlying the antioxidative effect of EPC-CM, changes in the activities of enzymes in the cellular antioxidative defense system were studied. The activities of SOD, catalase, and GPx were reduced by exposure to hydrogen peroxide in NHDF, and pretreatment with conditioned media protected the activities of these enzymes. Interestingly, while similar protective effects on SOD and GPX were observed for both conditioned media, EPC-CM increased catalase activity above normal level (Fig. 3a-c).

The mRNA and protein levels of antioxidant enzymes were also monitored. It was noticeable that, unlike the effects on enzyme activities, hydrogen peroxide upregulated mRNA 

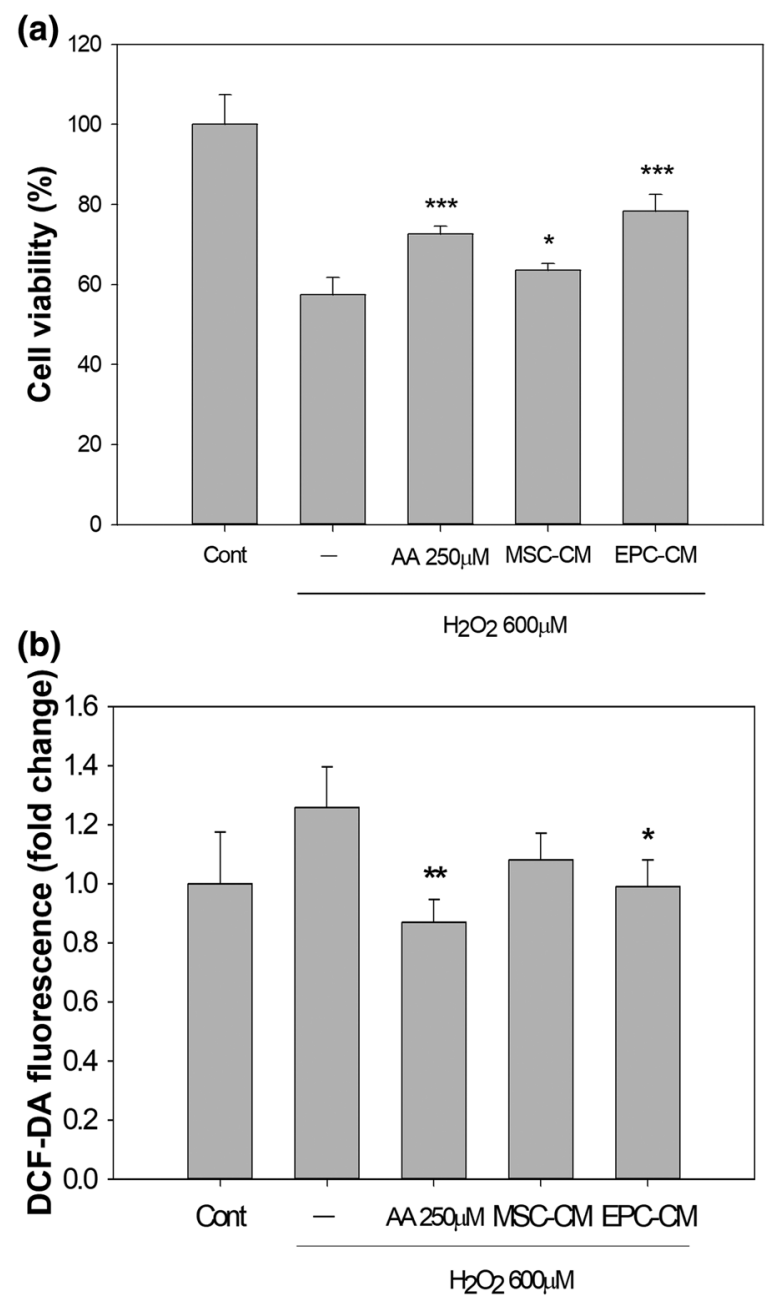

Fig. 2 Protection of NHDFs against oxidative stress due to hydrogen peroxide by conditioned media. Cells were treated with each conditioned medium prior to exposure to hydrogen peroxide. AA ascorbic acid. a AA and EPC$\mathrm{CM}$ increased the survival of fibroblasts under the influence of hydrogen peroxide. $\mathbf{b} \mathrm{AA}$ and EPC-CM reduced the production of intracellular $\operatorname{ROS.}{ }^{*} p<0.05$, ${ }^{* *} p<0.01$, and ${ }^{* * *} p<0.001$ versus control

expressions of SOD and GPX and suppressed catalase expression. Similar modifications were observed in protein levels of antioxidant enzymes by immunoblot assays, showing increased SOD and GPx, and decreased catalase levels by hydrogen peroxide treatment. Both EPC-CM and MSC-CM treatment normalized the expression of antioxidant enzymes in both mRNA and protein levels to that of untreated control cells (Fig. 3d, e).

\section{Modification of Collagen Biosynthesis}

Reduced collagen content is a marker in dermis of photoaged skin. Because ROS are important signals modifying collagen metabolism, the effect of conditioned media on collagen biosynthesis was studied. Type I procollagen C-terminal peptide in culture media was quantified by enzyme immunoassay as a measure of collagen biosynthesis in NHDF. Collagen biosynthesis was markedly reduced by hydrogen peroxide treatment, which was partially recovered by the action of $\mathrm{EPC}-\mathrm{CM}$ or MSC-CM. Type I procollagen mRNA expression levels were modified in parallel fashion with protein biosynthesis (Fig. 4a, b).

\section{Regulation of MAPK Signaling}

Phosphorylations of the members of MAPK signaling pathways were thought to be responsible for reduced collagen gene expression. Phosphorylations of Erk, JNK, c-Jun, p38, and Akt were stimulated by hydrogen peroxide treatment in NHDF. Both EPC-CM and MSC$\mathrm{CM}$ effectively inhibited hydrogen peroxideinduced phosphorylation of the proteins in MAPK cascade (Fig. 4c).

\section{Clinical Study}

A cosmetic essence containing 5\% EPC-CM was formulated and used in clinical study. Female volunteers $(n=25$, aged between 29 and 69) applied essence twice a day for 4 weeks. The signs of skin condition, including the depth of wrinkles at the outside of the eyes (crow's feet), the elevation of skin surface on the right cheek, and the texture small $(\mathrm{Ra})$ values of the right cheek, were improved significantly during clinical test period (Fig. 5a-d).

\section{DISCUSSION}

Solar ultraviolet (UV) is one of the major environmental factors that cause extrinsic aging of skin. It can cause wrinkles, oxidative stress, hyperpigmentation, and skin cancer $[25,26]$. 


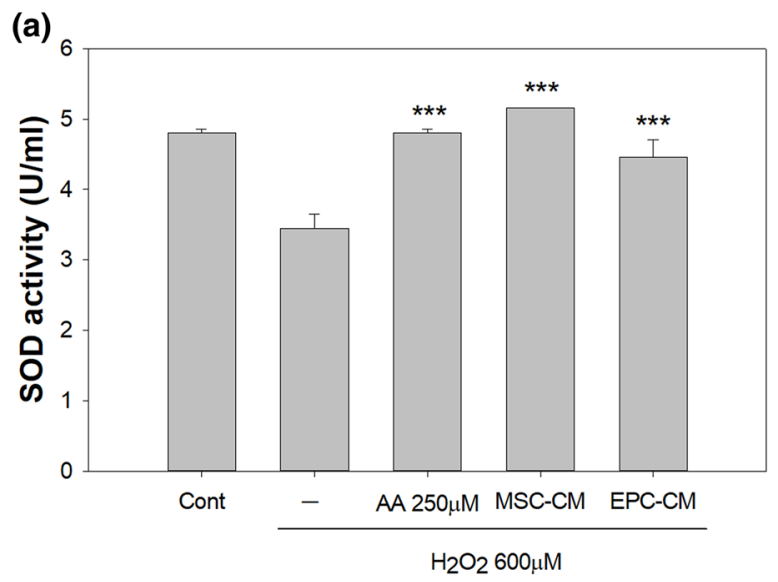

(d)
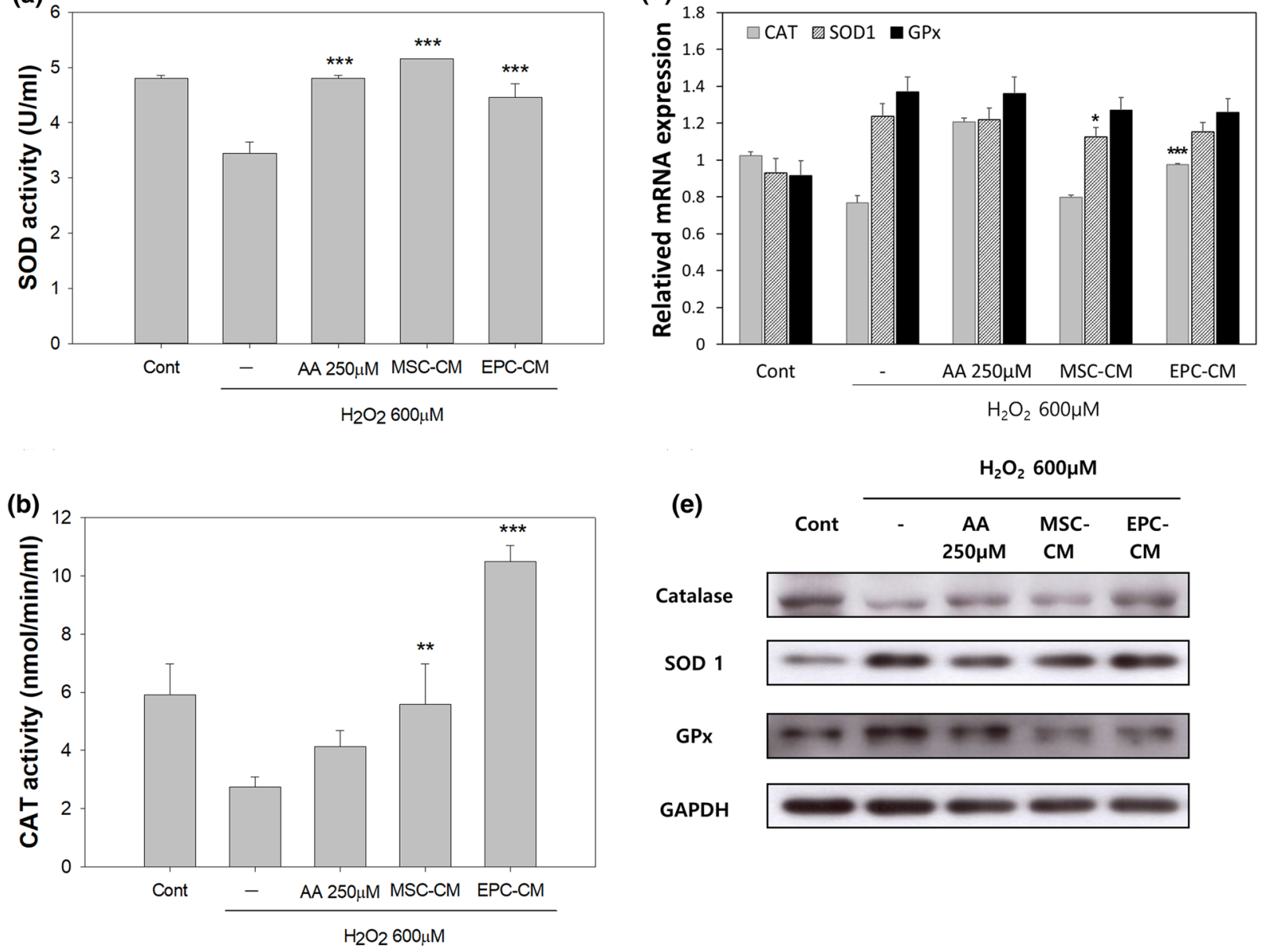

(e)

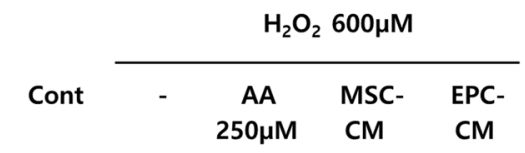

Catalase

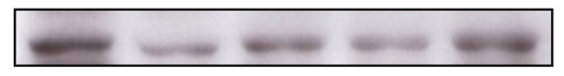

SOD 1

GPx
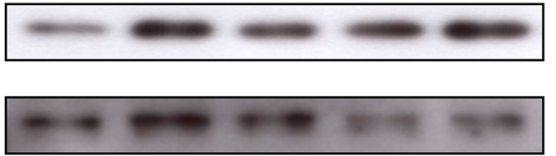

GAPDH

(c)

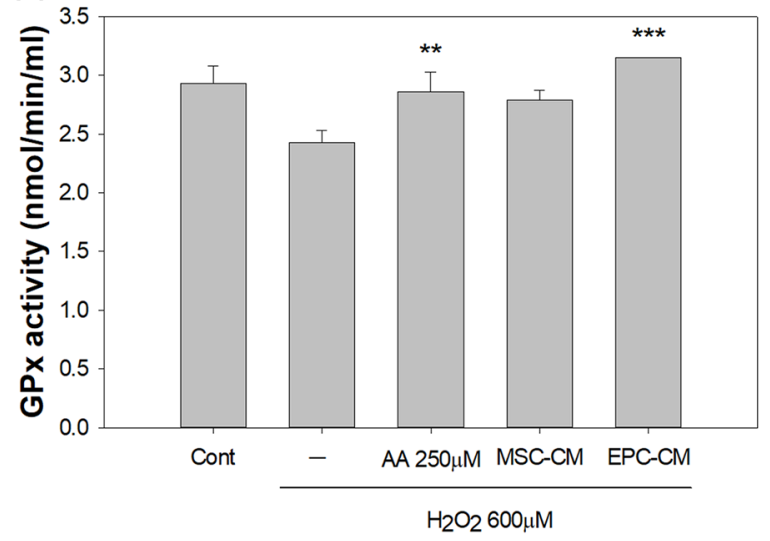

Fig. 3 Capacities of conditioned media to protect antioxidative defense enzymes from oxidative damage in NHDFs. Cells were treated with each conditioned medium prior to exposure to hydrogen peroxide. AA ascorbic acid. a-c AA

and each conditioned medium protected the loss of activities of SOD, catalase, and GPx. ${ }^{* *} p<0.01$, and ${ }^{* * *} p<0.001$ versus control. d, e mRNA and protein levels of SOD, catalase, and GPx 
(a)

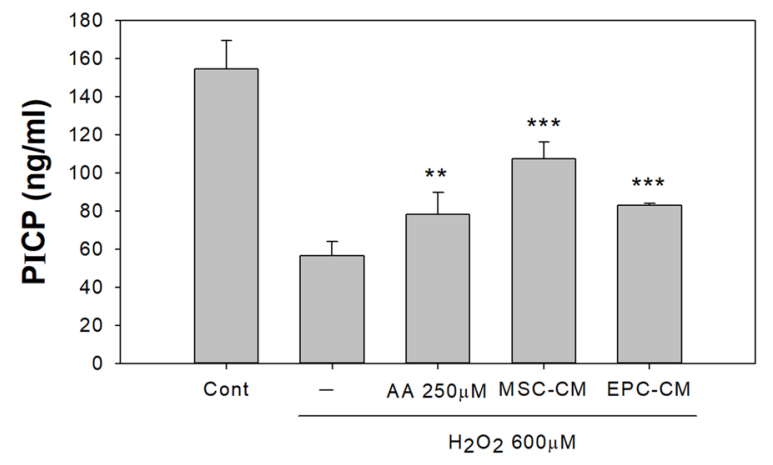

(b)

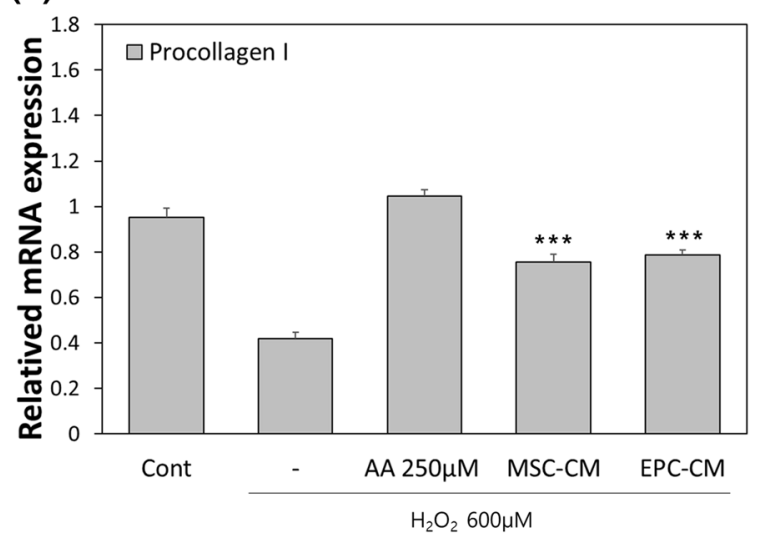

(c)

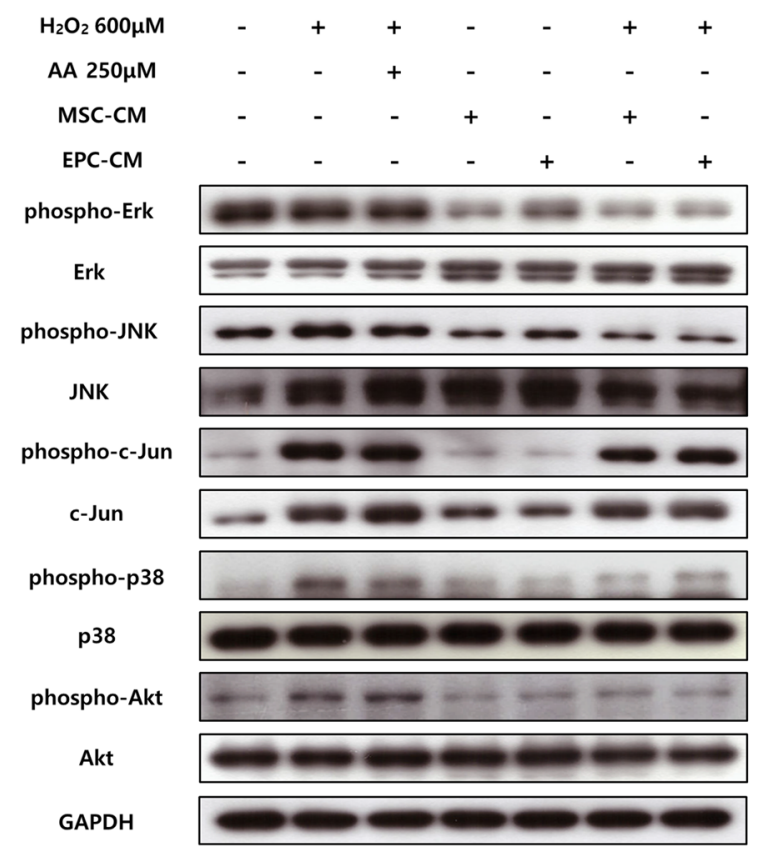

4 Fig. 4 Effect of conditioned media on collagen metabolism and MAPK signaling in NHDFs. Cells were treated with each conditioned medium prior to exposure to hydrogen peroxide. AA ascorbic acid. a ELISA analysis of secreted type I procollagen C-terminal peptide. ${ }^{* *} p<0.01$, and ${ }^{* * *} p<0.001$ versus control. $\mathbf{b}$ mRNA levels of type I procollagen. c Phosphorylation of MAPK signaling proteins

Various remedies have been used in anti-aging cosmetics, such as retinoids, small peptides including cytokines, and antioxidants from medicinal plants.

Recently, stem cells and their conditioned media were reported to show beneficial effects to improve signs of photoaging. It was reported that adipose-derived stem cell conditioned medium (ADSC-CM) shows antioxidant and anti-aging effects in human dermal fibroblasts $[27,28]$. Human placental multipotent mesenchymal stromal cell conditioned medium (hMSCs-CM) has inhibitory effects of oxidant and apoptosis [29]. Mesenchymal stem cells secreted cytokines and growth factors, such as vascular endothelial growth factor (VEGF), hepatocyte growth factor (HGF), and fibroblast growth factor (FGF), and these factors are reported to help defense against oxidative stress, accelerate wound healing, and modulate pigmentation in the skin [30-34]. Keratinocyte stem cells reside in the basal layer of epidermis and maintain the homeostasis of epidermis by supplying differentiating epidermal cells to upper layers. Keratinocyte stem cells also signal to surrounding tissues and cells via diffusible factors. Despite the crucial importance of keratinocyte stem cells in normal homeostasis of the skin, conditioned medium from keratinocyte stem cells has not been used frequently in cosmetics or related products, because keratinocyte stem cells readily undergo terminal differentiation when cultured and, therefore, it is difficult to establish stable culture of keratinocyte stem cells [18-20]. In this study, EPCs were obtained by differentiation from a mesenchymal stem cell line. Cultured in a specific medium containing hydrocortisone and ascorbic acid, hMSC was transdifferentiated to a 
(a)

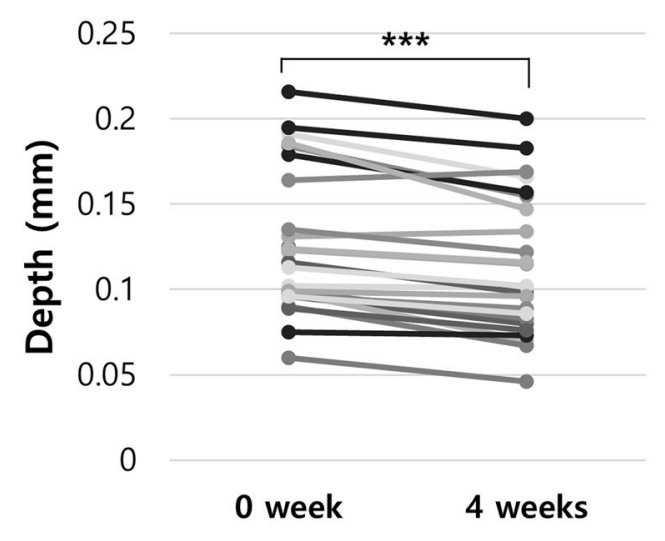

(c)

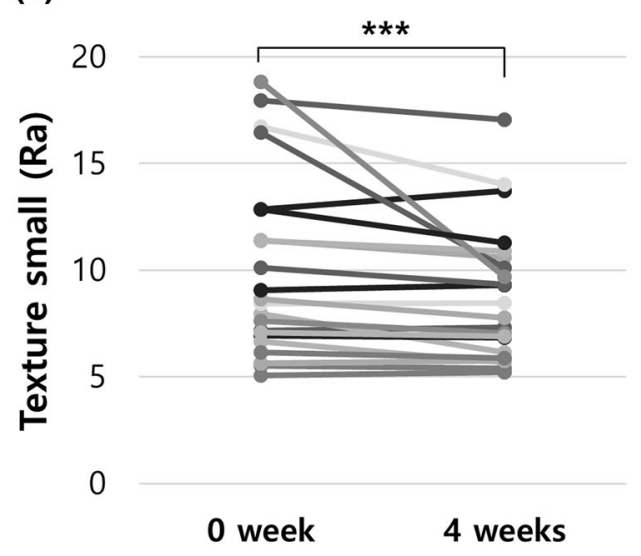

Fig. 5 Clinical results. A cosmetic essence containing 5\% EPC-CM was applied to the face of female volunteers $(n=25)$ twice a day for 4 weeks. ${ }^{* * *} p<0.001$ versus

cell with epidermal cell-like morphology which grew in cobblestone pattern clusters in monolayer culture and expressed markers for keratinocyte (involucrin and cytokeratins). Through this differentiation technique it is possible to produce EPC-CM in sufficient quantity with stable quality from stored mesenchymal stem cells.

Signs of aging are prominent in habitually sun-exposed skin, such as the face and neck, and reactive oxygen species are believed to be crucial factors leading to these changes $[25,35,36]$. When cultured human dermal fibroblasts are exposed to hydrogen peroxide, (b)

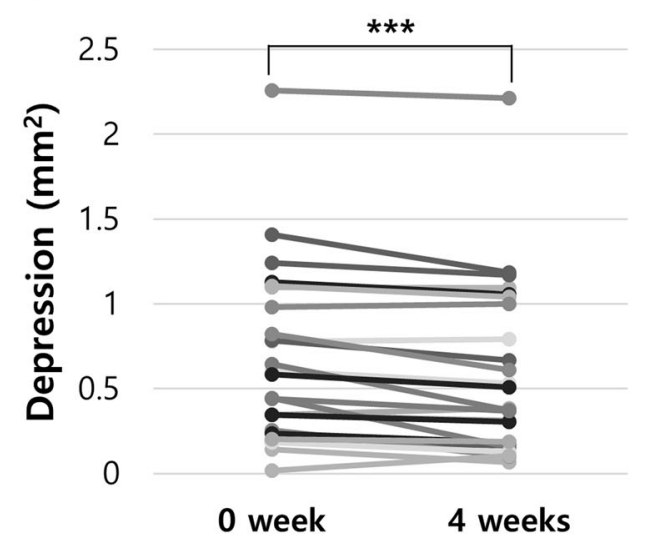

(d)

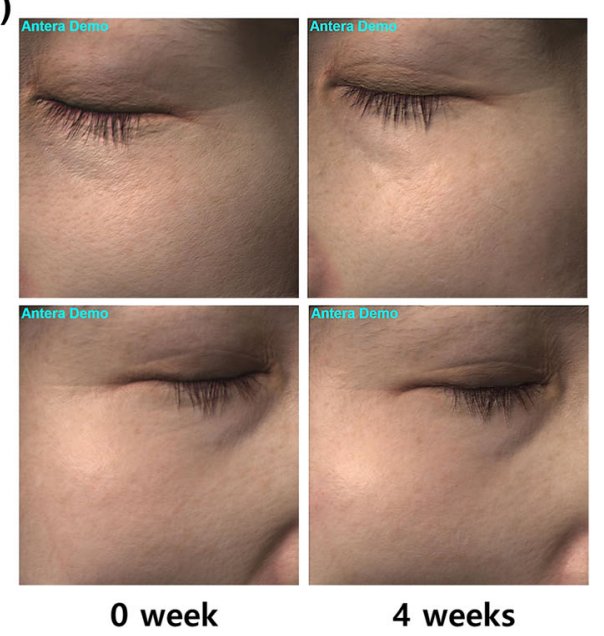

control. a Wrinkle (crow's feet). b Depression. c Skin texture. d Representative photographs

cell growth was inhibited and, as expected, cellular ROS level was elevated. The protective effect of EPC-CM on cell growth may be achieved by removing ROS from cells, as shown in low fluorescence levels in the DCFDA assay in cells which were pretreated with EPC-CM.

There are two possibilities to reduce intracellular ROS levels: direct scavenging of radical species or activation of the cellular antioxidative defense system. Because conditioned media showed little or no radical scavenging activities (data not shown), the effects of each conditioned medium on the activities of the cellular antioxidant system were studied. The 
intracellular ROS levels are regulated by altered production or by the cellular defense system composed of small antioxidant molecules and defensive enzymes, such as SOD, catalase, and GPx. When the balance of the oxidation/antioxidant system is broken, oxidative stress will occur in the cells of the skin. It was reported that UV irradiation reduced small molecular antioxidants, such as tocopherol and ascorbic acid, in skin equivalent cultures [37]. The alterations of defensive enzymes in NHDF induced by hydrogen peroxide were studied. The activities of SOD, catalase, and GPx were reduced by the action of hydrogen peroxide, and both EPC-CM and MSC-CM protected these enzymes from oxidative damage. These results might explain why conditioned media help cell growth by lowering ROS levels, which in turn was achieved by protection of antioxidant enzymes from oxidative stress. It is noticeable that, unlike enzyme activities, mRNA and protein levels of SOD and GPx were upregulated by hydrogen peroxide exposure. This response could be part of the cellular adaptation to acquire tolerance to cope with repeated oxidative stress, as previously reported by several investigators [38, 39]. The upregulated expression of SOD and GPx was also blocked by treatment with conditioned media.

Decreased collagen content in the dermis is one of the prominent signs of photoaging. UV light and ROS resulted in reduced synthesis of collagen types I and III, and elevated MMP-1 activity in dermis, and the signals of oxidative stress are mediated through phosphorylation of the proteins in the mitogen-activated protein kinase cascade, such as Erk, JNK, p38, and Akt [40-43]. Hydrogen peroxide exposure induced phosphorylation of MAPK signaling proteins and suppressed procollagen synthesis in NHDF. Both EPC-CM and MSC-CM, probably through antioxidative activities, prevented these downstream responses. It can be expected that these media have possibilities to improve signs of oxidative stress when applied on the skin and thus could be good candidates for anti-aging cosmetic ingredients.

Our results suggest that both EPC-CM and MSC-CM act in similar mode. EPC-CM, however, generally showed better protective effects over MSC-CM. To clarify the difference between these two media, secreted cytokines in the media were quantified and compared. Secretion of multiple cytokines into conditioned media was upregulated upon differentiation of hMSC to EPC (Table 2); these cytokines included TIMP1, TIMP2, TGF $\beta$ binding protein, and Smad4. UV or oxidative stress-induced activation of MAPK signaling, via AP-1 and NF- $\mathrm{BB}$, suppressed TGF $\beta$ receptor and Smad signaling proteins, which in turn reduced biosynthesis of procollagens in the dermis [44]. Enhanced TGF $\beta$ binding protein and Smad4 in EPC-CM may contribute to show stronger protective effects compared to MSC-CM. Elevated levels of TIMPs in EPC-CM also possibly help to prevent photoaging of the skin. Interestingly, nidogen-1 secretion was upregulated during differentiation to EPC. Nidogen-1 is an essential component of the basement membrane which operates as a linker molecule joining laminin and collagen IV networks [45, 46]. Recently, reduced nidogen-1, and poor basement membrane, was observed in aged skin and other tissues $[47,48]$. It was also reported that nidogen-1 supplementation enhanced basement membrane formation in a skin equivalent model [45].

For the evaluation of EPC-CM as a cosmetic ingredient, a cosmetic essence including 5\% EPC-CM was formulated and subjected to a clinical study. After 4 weeks of application period, parameters related to skin aging, such as wrinkle and skin texture, were significantly improved. Several reports demonstrated antiaging efficacies of stem cell conditioned media, mostly from stem cells which are not related to the skin, such as adipose-derived stem cells or placental cord blood stem cells $[29,49,50]$. This study is about the efficacy of conditioned media from stem cells with epidermal characteristics.

Although there are plenty of reports, including this study, showing the anti-aging properties of stem cell conditioned media, the precise mechanism is not clearly understood yet. Further studies are required to show which components are crucial among hundreds of secreted molecules, what the cellular targets of these molecules are, and how these molecules together elicit anti-aging efficacies. 


\section{CONCLUSIONS}

In this study, epidermal progenitor cells were differentiated from mesenchymal stem cells, and the anti-aging properties of EPC-CM were evaluated in NHDF and in a clinical study. EPCCM effectively protected NHDF from oxidative stress, and a cosmetic formulation containing EPC-CM significantly improved signs of aging.

\section{ACKNOWLEDGEMENTS}

The authors thank the participants of the study including volunteers for clinical study.

Funding. This work and article processing charges were supported by the Technology Innovation Program (or Industrial Strategic Technology Development Program, 10047890) funded by the Ministry of Trade, Industry \& Energy (MOTIE, Korea).

Authorship. All named authors meet the International Committee of Medical Journal Editors (ICMJE) criteria for authorship for this article, take responsibility for the integrity of the work as a whole, and have given their approval for this version to be published.

Disclosures. Su Ji Sohn, Ji Min Yu, Eun Young Lee, You Jin Nam, Jinwan Kim, Sukho Kang, Dong Hyun Kim, Aeri Kim, and Sangjin Kang have nothing to disclose.

Compliance with Ethics Guidelines. All procedures performed in studies involving human participants were in accordance with the ethical standards of the institutional research committee (IRB File No. 2016-04-039, Bundang CHA Hospital, Korea) and with the 1964 Declaration of Helsinki and its later amendments or comparable ethical standards. Informed consent was obtained from all individual participants included in the study. Additional informed consent was obtained from all individual participants for whom identifying information is included in this article.
Data Availability. The datasets generated and/or analyzed during the current study are available from the corresponding author on reasonable request.

Open Access. This article is distributed under the terms of the Creative Commons Attribution-NonCommercial 4.0 International License (http://creativecommons.org/licenses/ by-nc/4.0/), which permits any noncommercial use, distribution, and reproduction in any medium, provided you give appropriate credit to the original author(s) and the source, provide a link to the Creative Commons license, and indicate if changes were made.

\section{REFERENCES}

1. Gilchrest BA. Photoaging. J Invest Dermatol. 2013;133:E2-6.

2. Zouboulis CC, Adjaye J, Akamatsu H, Moe-Behrens G, Niemann C. Human skin stem cells and the ageing process. Exp Gerontol. 2008;43:986-97.

3. Abbas $\mathrm{O}$, Mahalingam M. Epidermal stem cells: practical perspectives and potential uses. Br J Dermatol. 2009;161:228-36.

4. Gnecchi M, Zhang Z, Ni A, Dzau VJ. Paracrine mechanisms in adult stem cell signaling and therapy. Circ Res. 2008;103:1204-19.

5. Chen S, Lewallen M, Xie T. Adhesion in the stem cell niche: biological roles and regulation. Development. 2013;140:255-65.

6. Kwon OS, Yoo HG, Han JH, Lee SR, Chung JH, Eun HC. Photoaging-associated changes in epidermal proliferative cell fractions in vivo. Arch Dermatol Res. 2008;300:47-52.

7. Yasui H, Sakurai H. Age-dependent generation of reactive oxygen species in the skin of live hairless rats exposed to UVA light. Exp Dermatol. 2003;12:655-61.

8. Black HS. Potential involvement of free radical reactions in ultraviolet light-mediated cutaneous damage. Photochem Photobiol. 1987;46:213-21.

9. Saitoh Y, Miyanishi A, Mizuno H, et al. Superhighly hydroxylated fullerene derivative protects human keratinocytes from UV-induced cell injuries together with the decreases in intracellular ROS 
generation and DNA damages. J Photochem Photobiol B. 2011;102:69-76.

10. Dringen R. Oxidative and antioxidative potential of brain microglial cells. Antioxid Redox Signal. 2005;7:1223-33.

11. Sohal RS, Allen RG. Oxidative stress as a causal factor in differentiation and aging: a unifying hypothesis. Exp Gerontol. 1990;25:499-522.

12. Patwardhan J, Bhatt P. Flavonoids derived from Abelmoschus esculentus attenuates UV-B induced cell damage in human dermal fibroblasts through Nrf2ARE pathway. Pharmacogn Mag. 2016;12:S129-38.

13. Zhan JY, Wang XF, Liu YH, et al. Andrographolide sodium bisulfate prevents UV-induced skin photoaging through inhibiting oxidative stress and inflammation. Mediat Inflamm. 2016;2016:3271451.

14. Baek B, Lee SH, Kim K, Lim HW, Lim CJ. Ellagic acid plays a protective role against UV-B-induced oxidative stress by up-regulating antioxidant components in human dermal fibroblasts. Korean J Physiol Pharmacol. 2016;20:269-77.

15. Walter MN, Wright KT, Fuller HR, MacNeil S, Johnson WE. Mesenchymal stem cell-conditioned medium accelerates skin wound healing: an in vitro study of fibroblast and keratinocyte scratch assays. Exp Cell Res. 2010;316:1271-81.

16. Kwon TR, Oh CT, Choi EJ, et al. Conditioned medium from human bone marrow-derived mesenchymal stem cells promotes skin moisturization and effacement of wrinkles in UVB-irradiated SKH-1 hairless mice. Photodermatol Photoimmunol Photomed. 2016;32:120-8.

17. Li M, Zhao $\mathrm{Y}$, Hao $\mathrm{H}$, et al. Umbilical cord-derived mesenchymal stromal cell-conditioned medium exerts in vitro antiaging effects in human fibroblasts. Cytotherapy. 2017;19:371-83.

18. Jones PH, Harper S, Watt FM. Stem cell patterning and fate in human epidermis. Cell. 1995;80:83-93.

19. Barrandon Y, Green H. Three clonal types of keratinocyte with different capacities for multiplication. Proc Natl Acad Sci USA. 1987;84:2302-6.

20. Jones PH, Watt FM. Separation of human epidermal stem cells from transit amplifying cells on the basis of differences in integrin function and expression. Cell. 1993;73:713-24.

21. Kim MJ, Shin KS, Jeon JH, et al. Human chorionicplate-derived mesenchymal stem cells and Wharton's jelly derived mesenchymal stem cells: a comparative analysis of their potential as placentaderived stem cells. Cell Tissue Res. 2011;346:53-64.

22. Carruthers A, Carruthers J, et al. A validated grading scale for crow's feet. Dermatol Surg. 2008;34:S173-8.

23. Jiang LI, Stephens TJ, Goodman R. SWIRL, a clinically validated, objective, and quantitative method for facial wrinkle assessment. Skin Res Technol. 2013;19:492-8.

24. Chavez-Munoz C, Nguyen KT, Xu W, Hong SJ, Mustoe TA, Galiano RD. Transdifferentiation of adipose-derived stem cells into keratinocyte-like cells: engineering a stratified epidermis. PLoS One. 2013;8:e80587.

25. Gilchrest BA. A review of skin ageing and its medical therapy. Br J Dermatol. 1996;135:867-75.

26. Chouinard N, Rouabhia M. Effects of all-trans retinoic acid on UVB-irradiated human skin substitute. J Cell Physiol. 1999;181:14-23.

27. Kim WS, Park BS, Sung JH, et al. Wound healing effect of adipose-derived stem cells: a critical role of secretory factors on human dermal fibroblasts. J Dermatol Sci. 2007;48:15-24.

28. Kim WS, Park BS, Park SH, Kim HK, Sung JH. Antiwrinkle effect of adipose-derived stem cell: activation of dermal fibroblast by secretory factors. J Dermatol Sci. 2009;53:96-102.

29. Liu SH, Huang JP, Lee RKK, et al. Paracrine factors from human placental multipotent mesenchymal stromal cells protect endothelium from oxidative injury via STAT3 and manganese superoxide dismutase activation. Biol Reprod. 2010;82:905-13.

30. Horwitz EM, Prather WR. Cytokines as the major mechanism of mesenchymal stem cell clinical activity: expanding the spectrum of cell therapy. Isr Med Assoc J. 2009;11:209-11.

31. Kim W-S, Park B-S, Kim H-K, et al. Evidence supporting antioxidant action of adipose-derived stem cells: protection of human dermal fibroblasts from oxidative stress. J Dermatol Sci. 2008;49:133-42.

32. Kim WS, Park SH, Ahn SJ, et al. Whitening effect of adipose-derived stem cells: a critical role of TGFbeta 1. Biol Pharm Bull. 2008;31:606-10.

33. Doorn J, Moll G, Le Blanc K, van Blitterswijk C, de Boer J. Therapeutic applications of mesenchymal stromal cells: paracrine effects and potential improvements. Tissue Eng Part B Rev. 2012;18:101-15. 
34. Liu Q, Luo Z, He S, et al. Conditioned serum-free medium from umbilical cord mesenchymal stem cells has anti-photoaging properties. Biotechnol Lett. 2013;35:1707-14.

35. Wulf HC, Sandby-Møller J, Kobayasi T, Gniadecki R. Skin aging and natural photoprotection. Micron. 2004;35:185-91.

36. Leyden JJ. Clinical features of ageing skin. Br J Dermatol. 1990;122(Suppl 35):1-3.

37. Podda M, Traber MG, Weber C, Yan LJ, Packer L. UV-irradiation depletes antioxidants and causes oxidative damage in a model of human skin. Free Radic Biol Med. 1998;24:55-65.

38. Seo YJ, Lee JW, Lee EH, Lee HK, Kim HW, Kim Y-H. Role of glutathione in the adaptive tolerance to $\mathrm{H}_{2} \mathrm{O}_{2}$. Free Radical Biol Med. 2004;37:1272-81.

39. Bose Girigoswami K, Bhaumik G, Ghosh R. Induced resistance in cells exposed to repeated low doses of $\mathrm{H}_{2} \mathrm{O}_{2}$ involves enhanced activity of antioxidant enzymes. Cell Biol Int. 2005;29:761-7.

40. Sun Z, Park SY, Hwang E, et al. Dietary Foeniculum vulgare Mill extract attenuated UVB irradiation-induced skin photoaging by activating of Nrf2 and inhibiting MAPK pathways. Phytomedicine. 2016;23:1273-84.

41. Wang Y, Chen H, Wang W, et al. N-terminal 5-mer peptide analog P165 of amyloid precursor protein inhibits UVA-induced MMP-1 expression by suppressing the MAPK pathway in human dermal fibroblasts. Eur J Pharmacol. 2014;734:1-8.

42. Hwang YP, Choi JH, Kim HG, et al. Cultivated ginseng suppresses ultraviolet B-induced collagenase activation via mitogen-activated protein kinases and nuclear factor kappaB/activator protein-1-dependent signaling in human dermal fibroblasts. Nutr Res. 2012;32:428-38.

43. Hwang YP, Kim HG, Han EH, et al. N-Acetylglucosamine suppress collagenases activation in ultraviolet B-irradiated human dermal fibroblasts: involvement of calcium ions and mitogen-activated protein kinases. J Dermatol Sci. 2011;63:93-103.

44. Kammeyer A, Luiten RM. Oxidation events and skin aging. Ageing Res Rev. 2015;21:16-29.

45. Nischt R, Schmidt C, Mirancea N, et al. Lack of nidogen-1 and -2 prevents basement membrane assembly in skin-organotypic coculture. J Invest Dermatol. 2007;127:545-54.

46. Kloepper JE, Tiede S, Brinckmann J, et al. Immunophenotyping of the human bulge region: the quest to define useful in situ markers for human epithelial hair follicle stem cells and their niche. Exp Dermatol. 2008;17:592-609.

47. Mondon P, Hillion M, Peschard O, et al. Evaluation of dermal extracellular matrix and epidermal-dermal junction modifications using matrix-assisted laser desorption/ionization mass spectrometric imaging, in vivo reflectance confocal microscopy, echography, and histology: effect of age and peptide applications. J Cosmet Dermatol. 2015;14:152-60.

48. Holland A, Dowling P, Zweyer M, et al. Proteomic profiling of cardiomyopathic tissue from the aged mdx model of Duchenne muscular dystrophy reveals a drastic decrease in laminin, nidogen and annexin. Proteomics. 2013;13:2312-23.

49. Son WC, Yun JW, Kim BH. Adipose-derived mesenchymal stem cells reduce MMP-1 expression in UV-irradiated human dermal fibroblasts: therapeutic potential in skin wrinkling. Biosci Biotechnol Biochem. 2015;79:919-25.

50. Li Q, Chen Y, Ma K, Zhao A, Zhang C, Fu X. Regenerative and reparative effects of human chorion-derived stem cell conditioned medium on photo-aged epidermal cells. Cell Cycle. 2016;15:1144-55. 